\title{
Du rêve des mangeurs de lumière
}

\section{Eberhard Wolff}

PD Dr rer. soc., membre de la rédaction Histoire de la médecine

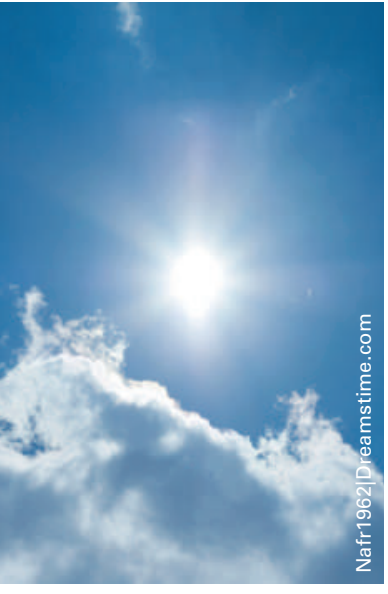

eberhard.wolff[at]saez.ch
Il s'agit là surtout d'un vieux rêve des végétariens. Pourquoi nous mammifères devons-nous écraser, engloutir et digérer d'autres êtres vivants, puis éliminer leurs résidus afin d'emmagasiner suffisamment de forces vitales? Qu'est-ce qui nous empêche de faire comme les plantes? Celles-ci puisent leur force vitale en exposant simplement leurs feuilles au soleil et en absorbant tout au plus un peu d'eau et quelques minéraux qu'elles trouvent dans le sol. Sans violence aucune. Mais: grâce à une biologiste, j'ai depuis déjà longtemps fait une croix sur l'idée d'un monde de plantes pacifico-socialistes. Nous devons nous imaginer la forme de vie qu'est la "symbiose» comme lutte perpétuelle plutôt que comme une sorte de car-sharing pacifiste de la nature. Mais mon objectif n'était pas de m'exprimer ici sur la manière dont les hommes usent de métaphores pour interpréter la nature.

Ainsi vit le vieux rêve des végétariens. En 2010, le réalisateur autrichien P.A. Straubinger a par exemple fait un documentaire sympathisant sur des personnes du monde entier qui prétendaient se nourrir (presque) exclusivement de lumière. A l'époque de la sortie du film, Bruno Kesseli publiait un article qui nous laissait une impression on ne peut plus délicieuse et nourrissante de ces mangeurs de lumière et de ce débat scientifique. Contrairement au film, l'article peut être consulté en ligne à n'importe quel moment [1].

Nous savons que notre corps s'essaie à la photosynthèse au moins pour la vitamine $\mathrm{D}$, mais cela ne va pas beaucoup plus loin. Mieux vaut néanmoins réfréner l'envie qui pourrait nous prendre de sourire de ces (supposés) mangeurs de lumière. Il est inutile et risqué de tirer de balles éclairantes quand on vit soi-même dans un palais de verre, surtout s'il est baigné de lumière. Le vieux rêve des avaleurs de lumière est bien plus répandu qu'il n'y paraît.

Le début du $\mathrm{XX}^{\mathrm{e}}$ siècle a été une époque marquée du fétichisme de la lumière, illustré par la randonnée en montagne ou les bains de soleil [2]. Leysin n'est pas seul à pouvoir chanter la manière dont la lumière est goulûment absorbée par les corps étendus sur les balcons du sanatorium. Quant aux citadins qui n'ont pas eu le privilège d'un séjour à Leysin, les sources de lumière UV artificielle n'auront pas nourri leur corps mais auront au moins essayé de lui apporter un peu d'énergie [3]. Cela se passait plutôt bien jusqu'à ce que les oncologues viennent nous gâcher quelque peu le plaisir.

Une variante largement répandue de l'alimentation solaire consiste en l'idée que nous n'absorbons pas la lumière à la place de la vraie nourriture, mais avec elle. Le birchermuesli en est, ou du moins en était un bel exemple, venu de Suisse. Max Bircher-Benner, le père du muesli, était d'avis (pour résumer sa pensée) que ses crudités étaient une "alimentation solaire» renfermant une énergie vitale spéciale issue de la lumière du soleil. Mais lorsque les crudités sont déjà cuites ou passées dans l'estomac d'une vache et transformées en viande, le rôti de bœuf et son accompagnement de légumes ne contiennent presque plus de cette saine énergie tirée de la lumière, seulement de méprisables calories. Très moderne, Bircher a bâti le fétichisme de la lumière autour d'une théorie physicaliste, celle de l'enseignement de l'entropie. A cette époque-là, c'était tout nouveau et plutôt tendance.

Un autre exemple, plus profane, de la manière dont nous absorbons la lumière du soleil avec la nourriture, m'a été rappelé par mon collègue cité plus haut, Tschofen. "Sunkist» était la boisson rêvée des écoliers dans les années 70. Le nom de cette coopérative d'agrumes californienne vient du mot "sun-kissed». Le rêve de l'alimentation solaire peut donc également être réalisé grâce à l'industrie.

La formule "à partir d'oranges mûries au soleil» est encore aujourd'hui une des favorites des publicitaires. Plus récemment: Rivella vend dans sa gamme de produits le jus «Michel», pas seulement le "Bodyguard", mais aussi le jus «Sunshine» avec la vitamine D, la «vitamine du soleil». L'ironie de la chose: si le corps est déjà capable de produire lui-même cette vitamine du soleil lors de bains de soleil, que vient faire la vitamine du soleil dans ce jus? J'imagine que "Sunshine», avec ses fruits délicats, n'étanche pas uniquement notre soif de jus mais rassasie aussi de manière conceptuelle notre inextinguible et symbolique faim de soleil.

De ce point de vue, nous sommes effectivement tous en quelque sorte des mangeurs de lumière qui nous en repaissons avec un plaisir évident, même si ce n'est pas uniquement calorique. Et me voilà reparti à m'interroger sur la manière dont les hommes usent de métaphores pour interpréter la nature.

\section{Références}

1 Kesseli B. «Prana» statt Müesli - oder doch nicht? Bull Méd Suisses. 2010;91(38):1494-6. www.saez.ch/docs/saez/archiv/ de/2010/2010-38/2010-38-827.pdf

2 Tschofen B (ed.). Piz Buin. Literarische Erkundungen 1865-2015. Bregenz: Bertolini; 2015.

3 Ingold N. Lichtduschen. Geschichte einer Gesundheitstechnik 1890-1975. Zurich: Chronos; 2015. 